\title{
Analytical Exact Solution of Neural Functional-differential Equations
}

\author{
Jamshad Ahmad ${ }^{1, *}$, Nida Fatima ${ }^{2}$ \\ ${ }^{1}$ Department of Mathematics, Faculty of Sciences, University of Gujrat, Pakistan \\ ${ }^{2}$ Department of Mathematics, National College of Business Administration and Economics, Pakistan
}

Copyright $(2016$ by authors, all rights reserved. Authors agree that this article remains permanently open access under the terms of the Creative Commons Attribution License 4.0 international License

\begin{abstract}
In this paper, we introduce a well-known technique Differential Transform method (DTM) which is very effective to control the convergence region of the approximate solution. The Differential Transform Method is applied to neutral functional-differential equation with proportional delays. The DTM produces an approximate solution with few hand computations without rounding off the error. Obtained solution of Neutral functional-differential equation with proportional delays reveals that DTM is one of the efficient and accurate methods.
\end{abstract}

Keywords Differential Transformation Method, Neutral Functional-differential Equation with Proportional Delays, Differential Equations

\section{Introduction}

The rapid development of nonlinear sciences observes number of new analytical and numerical methods. Most of these introduced techniques are of some inbuilt deficiencies including complicated and lengthy calculation, divergent results, limited convergence, small parameter assumption and non-compatibility with the physical nature of the problems. Recently the Variational Iteration Method (VIM) [6-7-8-9-10] has been applied to handle the nonlinear problems. Several modifications are available for the variational iteration method by Herisanu and Marinca[11] Noor and Mohyud-Din worked on strongly nonlinear problems[14], Yilmaz and Mustafa Inc [15], Zayed and Rahman [16], Geng and Cui [3], several researchers include Yilmaz and Mustafa Inc [15] modification has been caught much attention. In Refs. [17-20] Adomian's Decomposition Method (ADM), Homotopy Perturbation Method (HPM), Homotopy Analysis Method (HAM) and Variation of Parameter Method (VPM) are successfully applied to obtain the exact solution of differential equations.

In this study, we use the new developed analytical differential transform method (DTM) to solve the Neutral functional-differential equation with proportional delay. Firstly Zhou [21] introduced Differential transform Method on different type of nonlinear differential equations and has shown various remarkable results of this method. Using differential transformation method, a closed form series solution or an approximate solution can be obtained. The differential transform method obtains an analytical solution in the form of a polynomial. It is different from the traditional high order Taylor's series method, which requires symbolic competition of the necessary derivatives of the data functions. The Taylor series method is computationally expansive for large orders in terms of time. This method produced solutions in the form of polynomials and avoids large computational work and round off error. In present time, much nonlinear type of ODEs is easily solved by DTM .This method has been successfully applied to solve many types of nonlinear problems in science and engineering [22-24]. Recently, many adaptive numerical methods have been used which are very effective for these problems [26-29]. In this work the proposed DTM method is analytically applied to Neutral functional-differential equation with proportional delays. Several examples are given to verify the efficiency and compatibility of the proposed method.

Consider the following Neutral functional-differential equation with proportional delays:

$$
\begin{gathered}
\left(u(t)+a(t) u\left(p_{n}(t)\right)^{(n)}=\right. \\
\alpha u(t)+\sum_{i=0}^{n-1} b_{i}(t) u^{(i)}\left(p_{i}(t)\right)+g(t), t
\end{gathered}
$$

With initial conditions

$$
\sum_{i=0}^{n-1} C_{i j} u^{(i)}(0)=\beta_{j}, j=0,1,2 \ldots n-1 .
$$

Here, $a$ and $b_{i}(i=0,1,2 \ldots n-1)$ are given analytical functions, and $\alpha, p_{i}, C_{i j}, \beta_{j}$ are the given constants with $0<p_{i}<1(k=(0,1,2 \ldots n)$.

Neutral functional-differential equations with proportional delays represent a particular class of delay differential equation. Such functional-differential equations play an important role in the mathematical modeling of real world phenomena [1]. 
Wang et al. obtained approximate solutions for neutral delay differential equations by continuous Runge_Kutta methods [13] and one leg methods [12-13] Chen and Wang [2] applied original variational iteration method to compute approximate solutions for neutral delay differential equations. Obviously, most of these equations cannot be solved exactly. It is therefore necessary to design efficient numerical methods to approximate their solutions. The purpose of this paper is to apply DTM, to find the analytical solutions of Neutral functional-differential equations with proportional delays (1) along with (2), by demonstrating its ability and efficiency

\section{Analysis of Differential Transform Method}

Consider that the function $f(x)$ is continuously differentiable function on the interval $\left(x_{0}-r, x_{0}+r\right)$. The Differential Transform of the function $f(x)$ for the $k^{\text {th }}$ derivatives of the function is defined as

$$
F(k)=\frac{1}{k !}\left[\frac{d^{k} f(x)}{d x^{k}}\right]_{x=x_{0}}
$$

Where $f(x)$ is the original function and $F(k)$ is the transformed function.

The inverse differential transform $F(k)$ is defined as:

$$
f(x)=\sum_{k=0}^{\infty}\left(x-x_{0}\right)^{k} F(k)
$$

Put the value of $E q$. (3) into $E q$. (4), we get

$$
f(x)=\sum_{k=0}^{\infty}\left(x-x_{0}\right)^{k} \frac{1}{k !}\left[\frac{d^{k} f(x)}{d x^{k}}\right]_{x=x_{0}}
$$

The Basic operation of Differential Transformation can be constructed from Eq. (3) and Eq. (4) which is given below,

1. Theorem 1: If $f(x)=r(x) \pm p(x)$, then $F(k)=$ $R(k) \pm P(k)$,

2. Theorem 2: If $f(x)=\alpha r(x)$, then $F(k)=\alpha R(k)$,

3. Theorem 3: If $f(x)=\frac{d r(x)}{d x}$, then $F(k)=$ $(k+1) R(k+1)$,

4. Theorem 4: If $f(x)=\frac{d^{2} r(x)}{d x^{2}}$, then $F(k)=$ $(k+1)(k+2) R(k+2)$,

5. Theorem 5: If $f(x)=\frac{d^{n} r(x)}{d x^{n}}$, then $F(k)=$ $(k+1)(k+2) \ldots(k+n) R(k+n)$,

6. Theorem 6: If $f(x)=r(x) p(x)$, then $F(k)=$ $\sum_{l=0}^{k} P(l) R(k-l)$,

7. Theorem 7: If $f(x)=x^{n}$, then $F(k)=\delta(k-$ $n)$ where $\delta(k-n)\left\{\begin{array}{l}1, \text { if } k=n \\ 0, \text { if } k \neq n\end{array}\right\}$,

8. Theorem 8: If $f(x)=e^{\beta(x)}$, then $F(k)=\frac{(\beta)^{k}}{k !}$,

9. Theorem 9: if $f(x)=(1+x)^{n}$, then $F(k)=$ $\frac{n(n-1) \ldots(n-k+1)}{k !}$,

10. Theorem 10: If $f(x)=\sin (j x+\alpha)$, then $F(k)=$

$$
\frac{(j)^{k}}{k !}\left(\sin \left(\frac{\pi k}{2}+\alpha\right)\right)
$$

11. Theorem 11: If $f(x)=\cos (j x+\beta)$, then $F(k)=$ $\frac{(j)^{k}}{k !}\left(\cos \left(\frac{\pi k}{2}+\beta\right)\right)$,

12. Theorem 12: if $f(x)=r\left(\frac{x}{a}\right)$, then $F(k)=\frac{1}{a^{k}} R(k)$.

\section{Numerical Applications}

In this section we consider examples that show the efficiency of Differential Transform Method for solving Neutral functional-differential equations with proportional delays.

\section{Example 3.1}

Consider the first- order neutral functional-differential equation with proportional delay

$$
\begin{gathered}
u^{\prime}(t)=-u(t)+0.1 u(0.8 t)+0.5 u^{\prime}(0.8 t)+(0.32 t- \\
0.5) e^{-.8 t}+e^{-t},
\end{gathered}
$$

with initial condition

$$
u(0)=0 .
$$
(7),

Taking the Differential Transform of equation (6) and

$$
\begin{gathered}
(k+1)\left(1-\frac{0.5}{(0.8)^{k}}\right) U(k+1)=-U(k)+\frac{0.1}{(0.8)^{k}} U(k)+ \\
.32 \sum_{m=0}^{k} \frac{(-.8)^{m}}{l !} \delta(\mathrm{k}-\mathrm{m})-0.5\left(\frac{(-0.8)^{k}}{k !}\right)+\frac{(-1)^{k}}{k !} \\
U(0)=0
\end{gathered}
$$

The inverse differential Transform of $U(k)$ is defined as

$$
u(t)=\sum_{k=0}^{\infty} U(k) t^{k},
$$

Now substituting Eq. (9) into Eq. (8), we get the following values,

$$
\begin{aligned}
U(1)=1, U(2) & =-1, U(3)=\frac{1}{2 !}, U(4)=\frac{-1}{3 !}, U(5) \\
& =\frac{1}{4 !} \ldots
\end{aligned}
$$

Substitute all the values of $U(k)$ into Eq. (10), the following series solution will be obtained,

$$
\begin{gathered}
u(t)=\sum_{k=0}^{\infty} U(k) t^{k}=0+t-t^{2}+\frac{t^{3}}{2 !}-\frac{t^{4}}{3 !}+\frac{t^{5}}{4 !}-\cdots, \\
u(t)=t\left(1-t+\frac{t^{2}}{2 !}-\frac{t^{3}}{3 !}+\frac{t^{4}}{4 !}-\cdots\right),
\end{gathered}
$$

The closed form solution is $u(t)=t e^{-t}$.

\section{Example 3.2}

Consider the first- order homogenous neutral functional-differential equation with proportional delay.

$$
u^{\prime}(t)+u(t)-\frac{1}{2} u\left(\frac{t}{2}\right)-\frac{1}{2} u^{\prime}\left(\frac{t}{2}\right)=0,
$$


With initial condition

$$
u(0)=1 .
$$

Taking the Differential Transform of Eq. (11) and (12),

$$
(k+1)\left(1-\frac{1}{2.2^{k}}\right) U(k+1)=-U(k)+\frac{1}{2.2^{k}} U(k),
$$

with initial condition

$$
U(0)=1,
$$

The inverse differential Transform of $U(k)$ is defined as

$$
u(t)=\sum_{k=0}^{\infty} U(k) t^{k},
$$

Now substituting Eq. (14) into Eq. (13), we get the following values,

$$
U(1)=-1, U(2)=\frac{1}{2}, U(3)=\frac{-1}{3 !}, U(4)=\frac{1}{4 !} \ldots
$$

and so on. Substituting all the values of $U(k)$ into Eq. (15), the following series solution will be obtained,

$$
u(t)=\sum_{k=0}^{\infty} U(k) t^{k}=1-t+\frac{t^{2}}{2 !}-\frac{t^{3}}{3 !}+\frac{t^{4}}{4 !}-\frac{t^{5}}{5 !}+\cdots,
$$

The closed form solution is $u(t)=e^{-t}$.

\section{Example 3.3}

Consider the second order homogenous neutral functional-differential equation with proportional delay.

$$
\begin{gathered}
u^{\prime \prime}(t)-\frac{3}{4} u(t)-u\left(\frac{t}{2}\right)-u^{\prime}\left(\frac{t}{2}\right)-\frac{1}{2} u^{\prime \prime}\left(\frac{t}{2}\right)+t^{2}+t-1= \\
0,0 \leq t \leq 1
\end{gathered}
$$

with initial conditions

$$
u(0)=0, u^{\prime}(0)=0 .
$$

Taking the Differential Transform of Eq. (16) and (17),

$$
\begin{array}{r}
(k+1)(k+2)\left(1-\frac{1}{2.2^{k}}\right) U(k+2)=\frac{3}{4} U(k)+ \\
\frac{1}{2^{k}}(k+1) U(k+1)-\delta(k-2)-\delta(k-1)+\delta(k), \quad(18)
\end{array}
$$

with initial conditions

$$
U(0)=0, U(1)=0,
$$

The inverse differential Transform of $U(k)$ is defined as

$$
u(t)=\sum_{k=0}^{\infty} U(k) t^{k},
$$

Now substituting Eq. (19) into Eq. (18), we get the following values,

$$
U(2)=1, U(3)=0, U(4)=0
$$

and so on. Substituting all the values of $U(k)$ into Eq. (19), the following series solution will be obtained,

$$
u(t)=\sum_{k=0}^{\infty} U(k) t^{k}=0+0 t+t^{2}+0 t^{3}+0 t^{4} \ldots,
$$

The exact solution is $u(t)=t^{2}$.

\section{Example 3.4}

Consider the third order neutral functional-differential equation with proportional delay.

$$
\begin{gathered}
u^{\prime \prime \prime}(t)=u(t)+u^{\prime}\left(\frac{t}{2}\right)+u^{\prime \prime}\left(\frac{t}{3}\right)+\frac{1}{2} u^{\prime \prime \prime}\left(\frac{t}{4}\right)-t^{4}-\frac{t^{3}}{2}- \\
\frac{4}{3} t^{2}+21 t
\end{gathered}
$$

with initial conditions

$$
u(0)=0, u^{\prime}(0)=0, u^{\prime \prime}(0)=0 .
$$

Taking the Differential Transform of Eq. (21) and (22),

$$
\begin{gathered}
(k+1)(k+2)(k+3)\left(1-\frac{1}{2 \cdot 4^{k}}\right) U(k+3)=U(k)+ \\
\frac{1}{2^{k}}(k+1) U(k+1)+\frac{1}{3^{k}}(k+1)(k+2) U(k+2)- \\
\delta(k-4)-\frac{1}{2} \delta(k-3)-\frac{4}{3} \delta(k-2)+21 \delta(k-1),(23)
\end{gathered}
$$

with initial conditions,

$$
U(0)=0, U(1)=0, U(2)=0,
$$

The inverse differential Transform of $U(k)$ is defined as

$$
u(t)=\sum_{k=0}^{\infty} U(k) t^{k},
$$

Now substituting Eq. (24) into Eq. (23), we get the following values,

$$
U(3)=0, U(4)=1, U(5)=0 U(6)=0
$$

and so on. Substituting all the values of $U(k)$ into Eq. (25), the following series solution will be obtained,

$$
u(t)=\sum_{k=0}^{\infty} U(k) t^{k}=0+0 t+0 t^{2}+0 t^{3}+t^{4}+0 t^{5} \ldots
$$

The exact solution is $u(t)=t^{4}$.

\section{Example 3.5}

Consider the Logistic equation with proportional delay of the form [25] is

$$
u^{\prime}(t)=\rho u(t)(1-u(t-r)) t>0, \rho>0,
$$

with initial conditions

$$
u(0)=u_{0}=0.85, \rho=0.5, r=0 .
$$

Taking the Differential Transform of equation (26) and (27)

$(k+1) U(k+1)=0.5 U(k)-0.5 \sum_{l=0}^{k} U(l) U(k-l),(28)$

with initial conditions

$$
U(0)=0.85,
$$

The inverse differential Transform of $U(k)$ is defined as

$$
u(t)=\sum_{k=0}^{\infty} U(k) t^{k},
$$

Now substituting Eq. (29) into Eq. (28), we get the following values,

$$
U(1)=0.064, U(2)=-0.112, U(3)=-.00068
$$

and so on. Substituting all the values of $U(k)$ into Eq. (30), the following series solution will be obtained, 


$$
\begin{gathered}
u(t)=\sum_{k=0}^{\infty} U(k) t^{k} \\
=0.85+0.64 t-0.112 t^{2} \\
-.00068 t^{3} \ldots, \\
u(t)=\frac{u_{0}}{u_{o}+\left(1-u_{0}\right) e^{-\rho t}} .
\end{gathered}
$$

\section{Conclusions}

In this study, Differential Transform Method is extended successfully to solve analytical the neutral functional-differential equation with proportional delays. By applying DTM, we lucratively obtained the analytical approximate solution of neutral functional-differential equation with proportional delays. The obtained exact solutions revealed that the proposed method is easy to implement in finding the analytical solutions, accurate, fast and it reduce the size of the computational involvement. There is no need for calculating multiple integrals or derivatives, polynomials and less computational work is demanded compared to other popular methods. Differential Transform Method offer excellent opportunity for the future research.

\section{REFERENCES}

[1] Bellen, A. and Zennaro, M. Numerical methods for delay differential equations, Numerical Mathematics and Scientific Computation, Oxford University Press, New York, NY. (2003),

[2] Chen, X. and Wang, L. The variational iteration method for solving a neutral functional-differential equation with proportional delays, Computers and Mathematics with Applications, Vol. 59 No. 8, pp. 2696-702. (2010),

[3] Geng, F.Z. and Cui, M.G. Solving nonlinear multi-point boundary value problems by combining homotopy perturbation and variational iteration methods, International Journal of Nonlinear Sciences \& Numerical Simulation, Vol. 10, pp. 597-600. (2009)

[4] He, J.-H. Variational iteration method - A kind of non-linear analytical technique: some examples, International Journal of Non-Linear Mechanics, Vol. 34 No. 4, pp. 699-708.1999.

[5] [5] He, J.-H. Non-perturbative Methods for Strongly Nonlinear Problems, dissertation de-Verlag im Internet GmbH, Berlin. 2006.

[6] He, J.-H. Some asymptotic methods for strongly nonlinear equations, International Journal of Modern Physics B, Vol. 20 No. 10, pp. 1141-99.2006

[7] He, J.-H. Variational iteration method - some recent results and new interpretations, Journal of Computational and Applied Mathematics, Vol. 207 No. 1, pp. 3-17.2007

[8] He, J.-H. An elementary introduction to recently developed asymptotic methods and nanomechanics in textile engineering, International Journal of Modern Physics B, Vol. 22 No. 21, pp. 3487-578.2008

[9] He, J.-H. and Wu, X.-H. Variational iteration method: new development and applications, Computers \& Mathematics with Applications, Vol. 54, pp. 881-94.2007

[10] He, J.H., Wu, G.C. and Austin, F. The variational iteration method which should be followed, Nonlinear Science Letter a, Vol. 1, pp. 1-30.2010

[11] Herisanu, N. and Marinca, V. A modified variational iteration method for strongly nonlinear problems, Nonlinear Science Letter A, Vol. 1 No. 2, pp. 183-92. 2010

[12] Wang, W. and Li, S. On the one-leg methods for solving nonlinear neutral functional differential equations, Appl. Math. Comput., Vol. 193 No. 1, pp. 285-301.2007

[13] [13] Wang, W., Zhang, Y. and Li, S. Stability of continuous Runge Kutta-type methods for nonlinear neutral delay-differential equations, Appl. Math. Modell. Vol. 33 No. 8, pp. 3319-29 2009.

[14] Noor, M.A. and Mohyud-Din, S.T. Variational iteration method for solving higher-order nonlinear boundary value problems using He's polynomials, International Journal of Nonlinear Sciences \& Numerical Simulation, Vol. 9, pp. 141-56.2008

[15] Yilmaz, E. and Mustafa Inc. Numerical simulation of the squeezing flow between two infinite plates by means of the modified variational iteration method with an auxiliary parameter, Nonlinear Science Letter A, Vol. 1 No. 3, pp. 297-306. 2010.

[16] Zayed, E.M.E. and Rahman, H.M.A. On solving the KdV-Burger's equation and the $\mathrm{Wu}$ Zhang equations using the modified variational iteration method, International Journal of Nonlinear Sciences \& Numerical Simulation, Vol. 10, pp. 1093-103.2009.

[17] Ahmad, J., Mohyud-Din, Solving Fractional Vibrational Problem Using Restarted Fractional Adomian's Decomposition Method S.T., Life Sci J, 10(4), 210, 2013.

[18] Yıldırım, A., Koçak, H. Homotopy Perturbation Method for the Generalized Hirota-Satsuma Coupled KdV Equation Advances in Water Resources, 32(12), 1711, 2009.

[19] Ahmad, J., Bibi, Z., Noor, K. Application of Homotopy Perturbation Method to Nonlinear System of PDEs Journal of Science and Arts, 2(27), 131-138, 2014.

[20] Mohyud-Din,S.T., Noor, M.A., Waheed, A., World Applied Sciences Journal, 11(5), 622, 2010.

[21] Zhou,J.K., Differential Transformation and Its Applications for Electrical Circuits (in Chinese), Huazhong Univ. Press, 1986.

[22] Kangalgil, F., Ayaz, F.Solitary wave solutions for the KdV and $\mathrm{mKdV}$ equations by differential transform method, Chaos, Solitons and Fractals, 41, 464-472.2009.

[23] Ravi Kanth, A. S. V. and K. Aruna. Solution of singular two-point boundary value problems using differential transform method, Phys. Lett. A, Vol. 372(26), pp. 4671-4673.2008. 
[24] Ravi Kanth, A. S. V. and K. Aruna. Differential transform method for solving the linear and nonlinear Klein-Gordon equation, Comput. Phys.Commun., Vol. 180, pp. 708-711.2009.

[25] M.M. Khader. Numerical and theoretical treatment for solving linear and nonlinear delay differential equations using variational iteration method, Arab J Math Sci 19(2), 243-256.2012.

[26] B. Benhammouda, H. Vazquez-Leal, and L. Hernandez-Martinez Modified Differential Transform Method for Solving the Model of Pollution for a System of Lakes, Discrete Dynamics in Nature and Society, 2014,
[27] P. Das and V. Mehrmann, Numerical solution of singularly perturbed convection-diffusion- reaction problems with two small parameters, BIT Numerical Mathematics, doi:10.1007/s10543-015-0559-8,

[28] P. Das, Comparison of a priori and a posteriori meshes for singularly perturbed nonlinear parameterized problems, Journal of Computational and Applied Mathematics, 290, $16-25,2015$.

[29] P. Das and S. Natesan, Adaptive mesh generation for singularly perturbed fourth order ordinary differential equations, International Journal of Computer mathematics, 92(3), 562-578, 2015. 